\title{
Response to "Resolution of Dyssynchronous Left Ventricular Failure via Cardiac Resynchronization and Subsequent Radiofrequency Ablation in an Infant with Preexcitation"
}

\author{
Georgia Sarquella-Brugada $\cdot$ Josep Brugada
}

Published online: 29 September 2010

(C) Springer Science+Business Media, LLC 2010

In a recent article entitled Resolution of Dyssynchronous Left Ventricular Failure via Cardiac Resynchronization and Subsequent Radiofrequency Ablation in an Infant with Preexcitation, Ilina et al. [1] present the case of an infant with severe heart failure due to ventricular dyssynchrony provoked by a preexcitation. The patient initially was considered for transplantation but was epicardically paced 6 weeks later, leading to improvement of her clinical status. She was successfully ablated 5 years later, and the pacing device was explanted.

The article emphasizes the fact that ventricular preexcitation may produce a completely abnormal activation of the heart, leading to a pure electrical dyssynchrony, appropriately corrected in the reported case by left epicardial pacing. This important information should be considered as a possible physiopathologic explanation for any patient with preexcitation who presents with severe heart failure.

However, it is important to consider that implanting a pacemaker for false synchronization of the ventricle is not an adequate solution for this patient. Ablation of the accessory pathway should have been performed because this would have dealt with the origin of the problem. Ablation in small children is known to be technically difficult (probably not more difficult than implanting a device), but in experienced hands, it is safe and effective, similar to implanting a device [2].

G. Sarquella-Brugada ( $\square)$

Pediatric Arrhythmia Unit, Department of Cardiology,

Hospital Sant Joan de Déu, Barcelona, Spain

e-mail: georgia@brugada.org

J. Brugada

Pediatric Arrhythmia Unit, Department of Cardiology,

Hospital Sant Joan de Déu - Hospital Clínic, Barcelona, Spain
Placement of a device in an infant is not without risk, and subsequent complications for this palliative technique exist [3-5]. Performing an accessory pathway ablation also is not riskless. Exposing this patient to both risks is increasing the chances of complications and delaying the final and definitive solution: elimination of the electrical dyssynchrony produced by an accessory pathway.

\section{References}

1. Ilina MV, Abrams DJ, Lowe MD, Marek J (2010) Resolution of dyssynchronous left ventricular failure via cardiac resynchronization and subsequent radiofrequency ablation in an infant with preexcitation. Pediatr Cardiol 31:897-900

2. Sarquella-Brugada G, Brugada J (2010) Drug refractory arrhythmias: radiofrequency ablation in neoantes. In: 44th AEPC annual meeting, Innsbruck, May

3. Riede FT, Kostelka M, Dahnert I (2009) Cardiac strangulation: a rare but devastating complication of epicardial pacing causing progressive myocardial ischaemia. Eur Heart J 30:435

4. Silvetti MS, Drago F (2006) Upgrading of VVIR pacemakers with nonfunctional endocardial ventricular leads to VDD pacemakers in adolescents. Pacing Clin Electrophysiol 29:691-696

5. Watanabe H, Hayashi J, Sugawara M, Hashimoto T, Sato S, Takeuchi K (2000) Cardiac strangulation in a neonatal case: a rare complication of permanent epicardial pacemaker leads. Thorac Cardiovasc Surg 48:103-105 